\title{
Nanosecond-range imprint and retention characterized from polarization- voltage hysteresis loops in insulating or leaky ferroelectric thin films
}

\author{
A. Q. Jiang, ${ }^{1, a)}$ X. B. Liu, ${ }^{1}$ and Q. Zhang ${ }^{2}$ \\ ${ }^{1}$ State Key Laboratory of ASIC \& System, Department of Microelectronics, Fudan University, \\ Shanghai 200433, China \\ ${ }^{2}$ Department of Materials, Cranfield University, Cranfield, Bedfordshire, MK43 OAL, United Kingdom
}

(Received 22 August 2011; accepted 17 September 2011; published online 6 October 2011)

\begin{abstract}
We transferred ferroelectric domain switching currents under pulses into polarization-voltage $(P-V)$ hysteresis loops. With this transformation, it is possible to derive the remanent polarization and coercive voltage from domain switching currents after the shortest imprint and retention time of $35 \mathrm{~ns}$. After the separation of film leakage current from domain switching current, we measured the $P$ - $V$ hysteresis loop in a semiconducting $\mathrm{BiFeO}_{3}$ leaky thin film, where the apparent coercive field highly reaches $320 \mathrm{kV} / \mathrm{cm}^{2}$, suggestive of a different domain switching mechanism from other insulators. This technique facilitates nanosecond-range measurements of both ferroelectric capacitive and resistive memories. (C) 2011 American Institute of Physics. [doi:10.1063/1.3647577]
\end{abstract}

Nonvolatile ferroelectric random access memories make use of two switchable remnant polarization states of the materials to store binary information, which have advantages of low writing voltage, fast writing speed, and high endurance. ${ }^{1,2}$ Beyond the destructive readout of the information from capacitive ferroelectric memories, ${ }^{3}$ the nondestructive readout is possible from high- and low-conductance bipolar switching of ferroelectric resistive memories upon polarization reversal. ${ }^{4-6}$ However, the present commercial ferroelectric testers measure polarization-voltage $(P-V)$ hysteresis loops with a modified Sawyer-Tower circuit by applying a triangular/sinusoidal wave below $1 \mathrm{MHz}$ to an insulating thin film, which is different from memory writing/reading pulses with duration below $50 \mathrm{~ns}^{3}{ }^{3}$ Additionally, this technique is unable to differentiate the leakage current from the displacement current and, thus, unsuitable for the leaky film characterization. From pulse measurements, it is possible to separate the two currents and interweave the whole $P$ - $V$ loop from integrations of switching currents under many pulses in step-by-step increase of the voltage, ${ }^{4}$ which is nevertheless unsuitable for the fast reliability tests. Due to this consideration, it is necessary to transfer a positive/negative switching current into a $P-V$ hysteresis loop directly from which both the remanent polarization $\left(P_{\mathrm{r}}\right)$ and coercive voltage $\left(V_{\mathrm{c}}\right)$ can be predicted.

Insulating $\mathrm{Pb}\left(\mathrm{Zr}_{0.4} \mathrm{Ti}_{0.6}\right) \mathrm{O}_{3}$ (PZT) thin films were prepared using sol-gel spin coating on $\mathrm{IrO}_{2} / \mathrm{Pt} / \mathrm{TiO}_{2} / \mathrm{SiO}_{2} / \mathrm{Si}$ substrates with the thickness of $140 \mathrm{~nm}$. $\mathrm{Pt} / \mathrm{IrO}{ }_{2}$ top electrodes were sputtered on the films integrated into squares with side lengths of $1-200 \mu \mathrm{m}$. For the extraction of $P-V$ hysteresis loops in leaky films, oxygen-deficient (001) $\mathrm{BiFeO}_{3}$ (BFO) semiconducting films with the thickness of $270 \mathrm{~nm}$ were deposited on (100) $\mathrm{SrTiO}_{3}$ substrates at $650{ }^{\circ} \mathrm{C}$ with the $\mathrm{SrRuO}_{3}$ bottom electrode using pulsed laser deposition at the $\mathrm{O}_{2}$ pressure of $10 \mathrm{~Pa}^{4}$ Function-step voltage pulses with rising times of 5-7.5 ns were supplied by Agilent $33250 \mathrm{~A} / 81150 \mathrm{~A}$ pulse

\footnotetext{
${ }^{\text {a) }}$ Author to whom correspondence should be addressed. Electronic mail: aqjiang@fudan.edu.cn.
}

generators. Domain switching current $I_{\mathrm{sw}}$ with time $t$ under the pulse was monitored by an LC $6200 \mathrm{~A}$ oscilloscope in series with a ferroelectric capacitor $C_{\mathrm{f}}$. The total internal resistance of the pulse generator and oscilloscope is $R_{\mathrm{t}}=100 \Omega$.

There is a reference capacitor $C_{\text {ref }}\left(\gg C_{\mathrm{f}}\right)$ input in the Sawyer-Tower circuit in the characterization of $P-V$ loops under a triangular/sinusoidal wave. However, the characterization can be much faster if $C_{\text {ref }}$ is replaced by $R_{\mathrm{t}}$, as shown in the upper panel of Fig. 1. The voltage $V_{\mathrm{R}}$ across $R_{\mathrm{t}}$ is guarded by an oscilloscope with $I_{\mathrm{sw}}(t)=V_{\mathrm{R}}(t) / R_{\mathrm{t}}$. Figure 1 (a) shows $V_{\mathrm{R}}(t)$ for PZT with the electrode area of $S=4 \times 10^{-6}$ $\mathrm{cm}^{2}$ under bipolar pulses of $V(t)$ with the width of $30 \mathrm{~ns}$, where we observed two peaks of positive and negative domain switching. ${ }^{7}$ From the voltage of $V_{\mathrm{f}}(t)=V(t)-V_{\mathrm{R}}(t)$ across $C_{\mathrm{f}}$ and the polarization compensation charge of

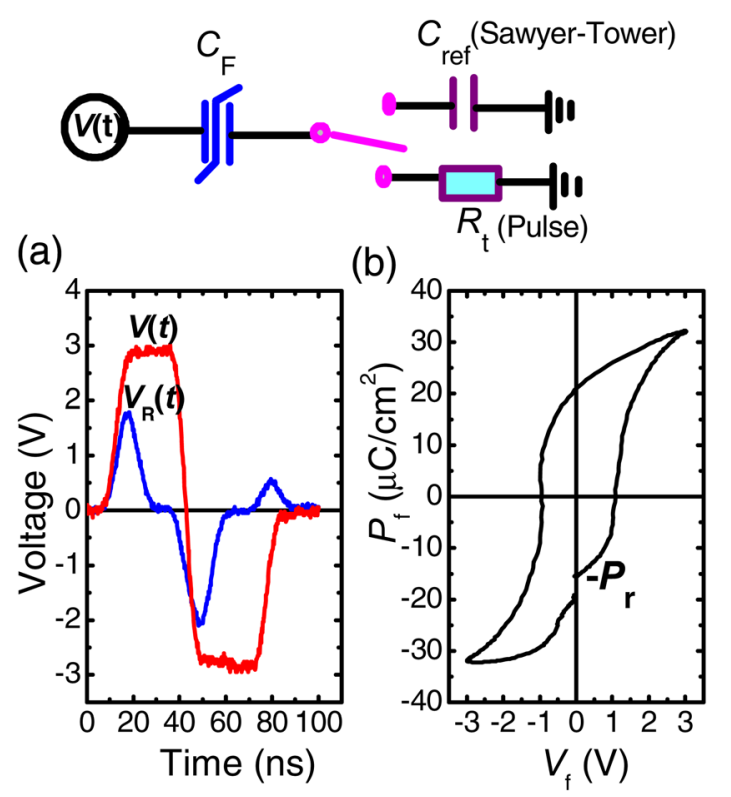

FIG. 1. (Color online) (a) The input pulse and voltage transient across $R_{\mathrm{t}}$ from the pulse measurement for PZT. (b) The transferred $P-V$ hysteresis loop from domain switching currents. The upper panel shows the equivalent circuit description of Sawyer-Tower and pulse measurements. 
$P_{f}(t)=\int_{0}^{t} \frac{V_{R}(t)}{S R_{t}} d t$ across $R_{\mathrm{t}}$, we can transfer the two currents into a $P_{\mathrm{f}}-V_{\mathrm{f}}$ hysteresis loop, as shown in Fig. 1(b).

For the integrated high-density memory, domain switching time decreases with the shrinkage of the capacitor area, ${ }^{8}$ as shown in Fig. 2. In contrast, $V_{\mathrm{c}}$ is no longer constant, which otherwise enhances with the area reduction. This is due to the fastened domain switching speed; the higher the domain switching speed, the larger the coercive voltage. ${ }^{9}$ The speed is believed to be proportional to the domain switching current density of $J_{\mathrm{sw}}=\left(V-V_{\mathrm{c}}\right) /\left(R_{\mathrm{t}} S\right)$, which is largely adjustable through $S$ spanning several orders of magnitude here.

The $P-V$ hysteresis loop shifts positively/negatively with time along the voltage axis after a negative/positive presetting pulse (imprint). ${ }^{10,11}$ The imprint is induced through the interfacial-layer charge injection between the film and the electrodes to build up an internal field, which is temporally uncompensated after polarization reversal and described by a semilogarithmic time dependence above an initial time $t_{0}$ on the order of several tens of seconds. ${ }^{10}$ Below $t_{0}$, the $V_{\mathrm{c}}$ shift is believed to be weak. However, with our improved measurements, we can largely shorten the imprint time into $35 \mathrm{~ns}$, as shown in Fig. 3(a), where the semilogarithmic time dependence for the positive/negative coercive voltage $\left( \pm V_{\mathrm{c}}\right)$ pertains true without the above $t_{0}$ limit, as shown by the solid-line fitting of the data. Meanwhile, we can measure the nanosecond-range ferroelectric depolarization, much faster than the time of seconds examined from the previous piezoresponse force microscopy, ${ }^{12}$ as shown in Fig. 3(b). $\left|-P_{\mathrm{r}}\right|$ decays exponentially with time, as shown by the solid-line fitting of the data, seemly like the interfacial-layer capacitor discharging until the depolarization field across the ferroelectric layer is smaller than the coercive field. ${ }^{8}$

If ferroelectric thin films are leaky for the resistive memories, $I_{\mathrm{sw}}(t)$ will show a leakage current level of $I_{\mathrm{L}} \neq 0$ after completion of domain switching in Fig. 4(a) (in solid lines). From a least-squares cubic polynomial fit of the $V_{\mathrm{f}}-I_{\mathrm{L}}$ dependence and the relationship of $V_{\mathrm{f}}(t)=V(t)$ - $I_{\mathrm{L}}(t) R_{\mathrm{t}}$, we can calculate $I_{\mathrm{L}}(V(t))$. ${ }^{4}$ After subtraction of $I_{\mathrm{L}}\left(V_{\mathrm{f}}(t)\right)$ from $I_{s w}(t)$, we obtained the domain displacement current only in Fig. 4(a) (in dashed lines), and thus the final

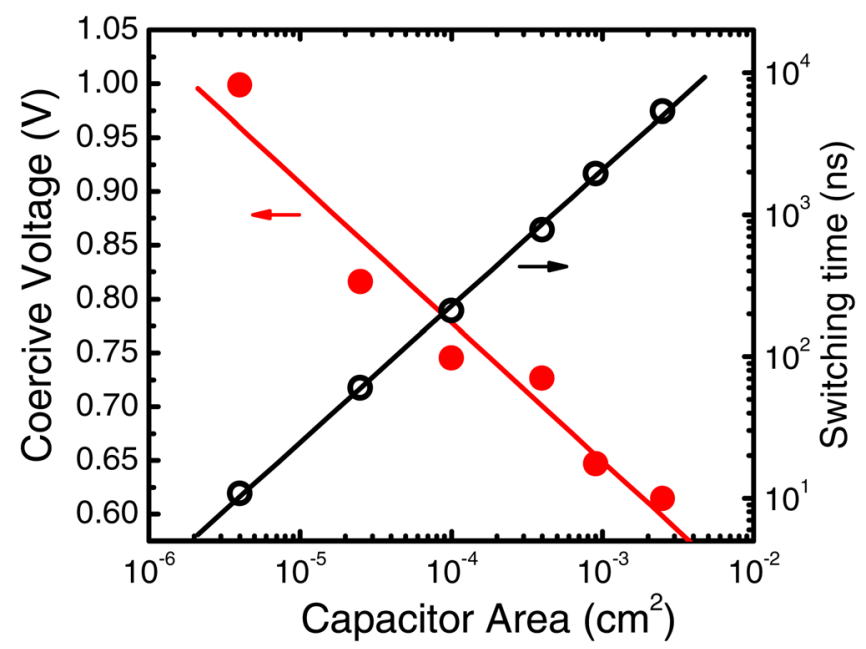

FIG. 2. (Color online) Capacitor area dependences of domain coercive voltage and switching time for PZT at $V=2.5 \mathrm{~V}$ fitted by two solid lines.
Pulse: $\quad t \quad 2.5 \mathrm{~V} / 35 \mathrm{~ns}$

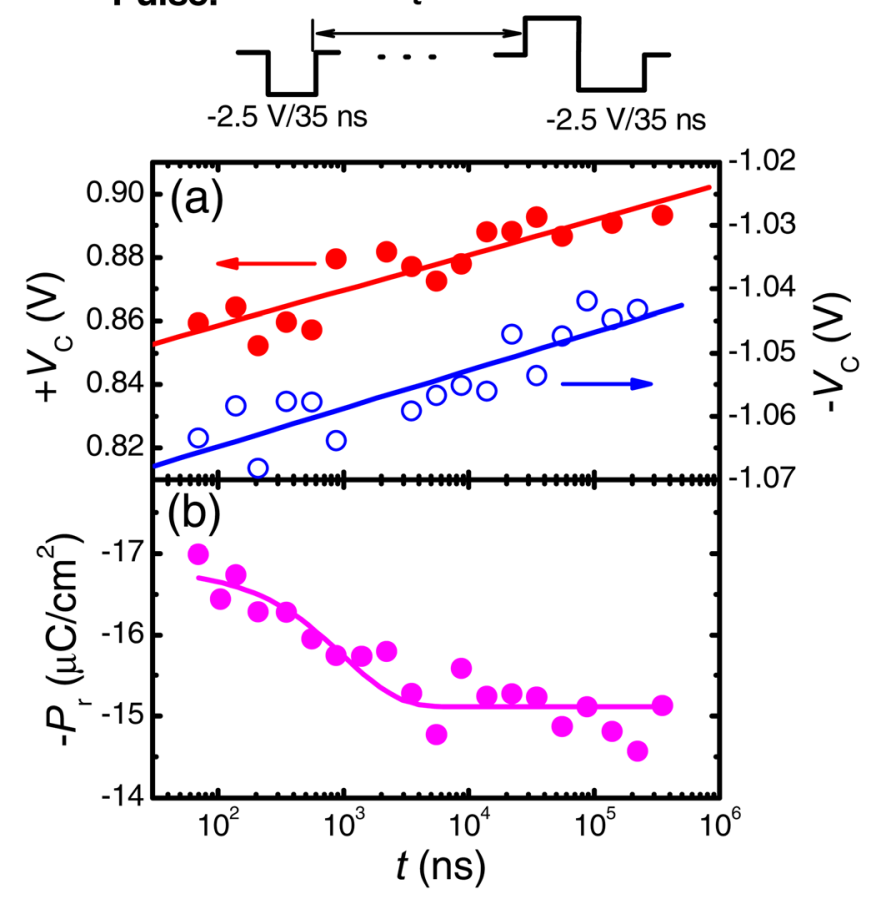

FIG. 3. (Color online) (a) Semilogarithmic time dependence of positive/ negative coercive voltage for PZT after the presetting pulse sketched in the upper panel, where the solid lines are the best fitting of the data. (b) Exponential degradation of the remanent polarization with time fitted by the solid line.

$P_{\mathrm{f}}-V_{\mathrm{f}}$ loop with $P_{\mathrm{r}}=61-64 \mu \mathrm{C} / \mathrm{cm}^{2}$ in Fig. 4(b), in agreement with (001) oriented BFO insulators. ${ }^{13}$

The square loops in Fig. 4(b) are unavailable from the commercial tester (Radiant Premier II) using a modified Sawyer-Tower circuit, as shown by the opened symbols, and also different from the loop interweaved with switching current integrations under many pulses. ${ }^{4}$ Unlike ferroelectric insulators, the coercive field here is around $320 \mathrm{kV} / \mathrm{cm}$ at $V_{\mathrm{f}}=12 \mathrm{~V}$ in Fig. 4(b), which nevertheless reduces quickly with the $V_{\mathrm{f}}$ reduction. This suggests the domain switching mechanism in a semiconductor different from those in other
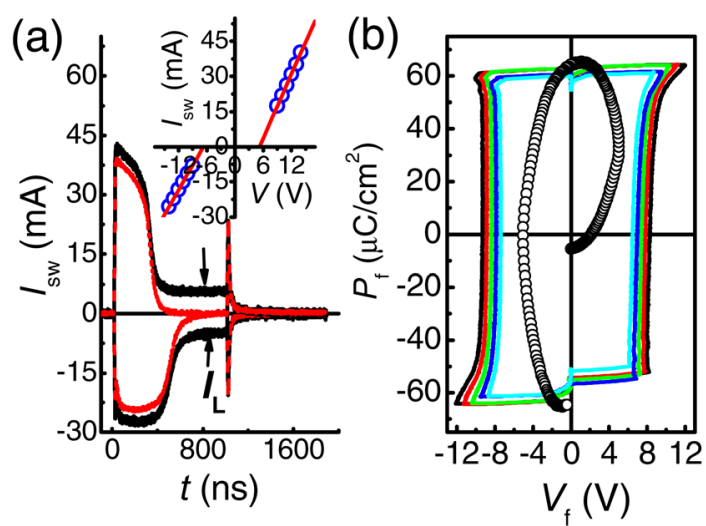

FIG. 4. (Color online) (a) Positive/negative domain switching current transient for BFO with an electrode area of $8.35 \times 10^{-5} \mathrm{~cm}^{2}$ at $V= \pm 12 \mathrm{~V}$ and pulse width of $1000 \mathrm{~ns}$ before (a solid line) and after (a dashed line) leakage current deduction, where the inset shows the voltage dependence of domain switching current fitted by two solid lines. (b) The transferred $P-V$ hysteresis loops from domain switching currents, where the opened symbols show the measurement from a Radiant tester at $50 \mathrm{kHz}$. 
insulators. ${ }^{14}$ Equivalent circuit description of this mechanism can assume the presence of a large contact resistance $R_{\mathrm{c}}$ between the film and electrodes, ${ }^{15}$ which is only $6.2 \Omega$ in PZT. The apparent coercive voltage in the loop consists of the intrinsic coercive voltage of domain switching and the voltage of $I_{\mathrm{sw}} R_{\mathrm{c}}$ across $R_{\mathrm{c}}$, where $I_{\mathrm{sw}}=\left(V-V_{\mathrm{c}}\right) /\left(R_{\mathrm{c}}+R_{\mathrm{t}}\right)^{4,8}$ From the linear dependence of domain switching current on $V$ shown by the inset in Fig. 4(a), we got the intrinsic $\pm V_{\mathrm{c}}=+5.17 \mathrm{~V} /-6.74 \mathrm{~V}$ with asymmetric $R_{\mathrm{c}}=121 \Omega / 183 \Omega$ in $\mathrm{BFO},{ }^{15}$ in agreement with the coercive field of $\sim 200 \mathrm{kV} /$ $\mathrm{cm}$ in other insulators. ${ }^{13}$

In conclusion, we estimated $P_{\mathrm{r}}$ and $V_{\mathrm{c}}$ after domain switching current transformation into a $P-V$ hysteresis loop with pulse width as short as $30 \mathrm{~ns}$. After deduction of the leaky current from the switching current, we extracted the $P$ $V$ hysteresis loop in a leaky BFO thin film with domain switching mechanism different from other insulators. This technique is unique and superior to traditional SawyerTower measurements for the nanosecond characterization of imprint and retention in either insulating or leaky thin films.

This work was supported by the Shanghai Key Program (1052nm07600) and the Program for Professor of Special Appointment (Eastern Scholar) at Shanghai.

${ }^{1}$ J. F. Scott, Ferroelectric Memories (Springer, Heidelberg, Germany, 2000), pp. 24-50.
${ }^{2}$ H. Kohlstedt, Y. Mustafa, A. Gerber, A. Petraru, M. Fitsilis, R. Meyer, U. Böttger, and R. Waser, Microelectron. Eng. 80, 296 (2005).

${ }^{3}$ Y. Shimojo, A. Konno, J. Nishimura, T. Okada, Y. Yamada, S. Kitazaki, H. Furuhashi, S. Yamazaki, K. Yahashi, K. Tomioka, Y. Minami, H. Kanaya, S. Shuto, K. Yamakawa, T. Ozaki, H. Shiga, T. Miyakawa, S. Shiratake, D. Takashima, I. Kunishima, T. Hamamoto, and A. Nitayama, in Proceedings of the Symposium on VLSI Technology (Japan Society Applied Physics, Tokyo, 2009), pp. 218-219.

${ }^{4}$ A. Q. Jiang, C. Wang, K. J. Jin, X. B. Liu, J. F. Scott, C. S. Hwang, T. A. Tang, H. B. Lu, and G. Z. Yang, Adv. Mater. 23, 1277 (2011).

${ }^{5}$ V. Garcia, S. Fusil, K. Bouzehouane, S. Enouz-Vedrenne, N. D. Mathur, A. Barthelemy, and M. Bibes, Nature 460, 81 (2009).

${ }^{6}$ P. Maksymovych, S. Jesse, P. Yu, R. Ramesh, A. P. Baddorf, and S. V. Kalinin, Science 324, 1421 (2009).

${ }^{7}$ A. Gruverman, D. Wu, and J. F. Scott, Phys. Rev. Lett. 100, 097601 (2008).

${ }^{8}$ A. Q. Jiang, Y. Y. Lin, and T. A. Tang, J. Appl. Phys. 101, 104105 (2007).

${ }^{9}$ A. Q. Jiang, H. J. Lee, C. S. Hwang, and J. F. Scott, "Sub-picosecond processes of ferroelectric domain switching from field and temperature experiments," Adv. Funct. Mater. (in press).

${ }^{10}$ A. K. Tagantsev, I. Stolichnov, and N. Setter, J. Appl. Phys. 96, 6616 (2004).

${ }^{11}$ W. L. Warren, H. N. Al-Shareef, D. Dimos, and B. A. Tuttle, Appl. Phys. Lett. 68, 1681 (1996).

${ }^{12}$ C. S. Ganpule, V. Nagarajan, S. B. Ogale, A. L. Roytburd, E. D. Williams, and R. Ramesh, Appl. Phys. Lett. 77, 3275 (2000).

${ }^{13}$ R. R. Das, D. M. Kim, S. H. Baek, C. B. Eom, F. Zavaliche, S. Y. Yang, R. Ramesh, Y. B. Chen, X. Q. Pan, X. Ke, M. S. Rzchowski, and S. K. Streiffer, Appl. Phys. Lett. 88, 242904 (2006).

${ }^{14}$ P. Maksymovych, S. Jesse, M. Huijben, R. Ramesh, A. Morozovska, S. Choudhury, L. Q. Chen, A. P. Baddorf, and S. V. Kalinin, Phys. Rev. Lett. 102, 017601 (2009).

${ }^{15}$ H. J. Lee, G. H. Kim, M. H. Park, A. Q. Jiang, and C. S. Hwang, Appl. Phys. Lett. 96, 212902 (2010). 